\title{
A CLASS OF ABSOLUTE RETRACTS IN SPACES OF INTEGRABLE FUNCTIONS
}

\author{
ALBERTO BRESSAN, ARRIGO CELLINA, AND ANDRZEJ FRYSZKOWSKI
}

(Communicated by Kenneth R. Meyer)

\begin{abstract}
We consider a class of subsets of $L^{1}$, that are shown to be absolute retracts, that contains at once decomposable sets and sets of solutions to Lipschitzean differential inclusions. In this way we generalize and unify a number of different previous results.
\end{abstract}

\section{INTRODUCTION}

Let $E$ be a Banach space and consider a multifunction $\phi: E \rightarrow 2^{E}$ with closed, bounded values, that is contractive with respect to the Hausdorff metric $d_{H}$, i.e.,

$$
d_{H}(\phi(u), \phi(v)) \leq \alpha \cdot|u-v|
$$

for some $\alpha<1$ and all $u, v \in E$.

If the values of each $\phi(u)$ are convex, then the set of fixed points $\mathscr{F}=$ $\{u: \phi \in \phi(u)\}$ is known to be an absolute retract [9]. In this paper we prove that the same is true when $E=L^{1}(T)$ for some measure space $T$ and the values of $\phi(u)$ are decomposable. This set $\mathscr{F}$ is, in general, not decomposable, while every decomposable set is the set of fixed points of a constant map. The primary motivation for the present study comes from differential inclusions. Let $F:[0, T] \times R^{n} \rightarrow 2^{R^{n}}$ be a Lipschitz continuous multifunction with compact, not necessarily convex, values. Call $S_{\xi}$ the set of all Carathéodory solutions of the problem:

$$
\dot{x} \in F(t, x), \quad x(0)=\xi .
$$

Then the set of derivatives $\mathscr{F}_{\xi}=\left\{\dot{u}: u \in S_{\xi}\right\}$ can be represented as the set of fixed points of contractive multifunction with decomposable values, related to the Picard operator. On the other hand, every bounded decomposable set on $[0, T]$ is the set of derivatives of the solutions to $x^{\prime}(t) \in F(t)$. Our result implies that $\mathscr{F}_{\xi}$ is an absolute retract of $L^{1}\left([0, T], R^{n}\right)$. Moreover, one can construct retractions that depend continuously on the initial data $\xi$. This

Received by the editors September 20, 1989 and, in revised form, April 7, 1990.

1980 Mathematics Subject Classification (1985 Revision). Primary 34A60, 49A50, 49E15, $54 \mathrm{C} 15,54 \mathrm{C} 20$. 
provides a unified and abstract approach to several results concerning approximations and continuous selections for solutions of Lipschitz continuous differential inclusions (see $[3-5,8]$ ). As a corollary we obtain the acyclicity and the fixed point property for every set $\mathscr{F}_{\xi}$.

\section{THE MAIN RESULT}

Let $T$ be a measure space with a finite, positive, nonatomic measure $\mu$. Given a Banach space $E$, let $L^{1}=L^{1}(T, E)$ be the Banach space of all Bochner $\mu$-integrable functions $u: T \rightarrow E$ with the norm $\|u\|=\int_{T}|u(t)| d \mu$, where $|\cdot|$ stands for the norm in $E$ (see [10]).

We assume that $L^{1}(T, E)$ is separable. For further notations and definitions we refer to [7]. We recall that $d_{H}$ stands for the Hausdorff distance and that for a given family $V$ of real functions, the map $T(t)=\operatorname{ess} \inf \{v(\cdot): v \in V\}$ is the largest measurable lower bound of $V$. A subset $K \subset L^{1}$ is called decomposable if for every $u, v \in K$ and every measurable $A \subset T$,

$$
u \cdot \chi_{A}+v \cdot \chi_{T \backslash A} \in K \text {. }
$$

Denote by $D$ the family of all nonempty, bounded, closed, and decomposable subsets of $L^{1}$ and consider a family of maps

$$
\phi_{\lambda}: L^{1} \rightarrow D
$$

depending on a parameter $\lambda$ from a separable metric space $\Lambda$.

Theorem 1. Let $(\lambda, u) \rightarrow \phi_{\lambda}(u)$ be a continuous map from $\Lambda \times L^{1}$ into $D$, that is contractive with respect to $u$, i.e.,

$$
d_{H}\left(\phi_{\lambda}(u), \phi_{\lambda}(v)\right) \leq \alpha\|u-v\|
$$

for some $\alpha<1$, all $u, v \in L^{1}$, and any $\lambda \in \Lambda$. Then each set of fuxed points

$$
\mathscr{F}_{\lambda}=\left\{u: u \in \phi_{\lambda}(u)\right\}
$$

is an absolute retract. A retraction can be chosen that depends continuously on $\lambda$; namely, there exists a continuous map $g: \Lambda \times L^{1} \rightarrow L^{1}$ such that:

$$
\begin{array}{cl}
g(\lambda, u) \in \mathscr{F}_{\lambda} & \text { for all } u \in L^{1}, \\
g(\lambda, u)=u & \text { for all } u \in \mathscr{F}_{\lambda} .
\end{array}
$$

Proof. Choose $\delta>0$ so small that

$$
\alpha(1+\delta \cdot \mu(T))<1
$$

and let

$$
\Psi_{\lambda}(u)=\operatorname{ess} \inf \left\{|u(\cdot)-v(\cdot)|: v \in \phi_{\lambda}(u)\right\} .
$$

Denote by $O \subset \Lambda \times L^{1}$ the set

$$
O=\left\{(\lambda, u): u \notin \phi_{\lambda}(u)\right\}
$$


and observe that $O$ is open. For each $(\lambda, u) \in O$ define

$$
k_{\lambda}(u)(t)=\Psi_{\lambda}(u)(t)+\delta\left(1 \wedge \int_{T} \Psi_{\lambda}(u)(s) d \mu(s)\right)
$$

and

$$
K_{\lambda}(u)=\operatorname{cl}\left\{v \in \phi_{\lambda}(u):|u(t)-v(t)|<k_{\lambda}(u)(t) \text { a.e. in } T\right\},
$$

where cl denotes the closure in $L^{1}$.

Notice that from Proposition 2 in [2] it follows that $K_{\lambda}(u) \in D$. Thus from Proposition 3 in [2] the map $(\lambda, u) \rightarrow K_{\lambda}(u)$ is 1.s.c. from $O$ into $D$, since $(\lambda, u) \rightarrow k_{\lambda}(u)$ is continuous on $O$. By the selection theorem due to Bressan and Colombo [2, Theorem 3] there exists a continuous selection $f(\lambda, u) \in K_{\lambda}(u)$ defined on $O$. Extend $f$ to $\Lambda \times L^{1}$ by setting

$$
f(\lambda, u)=u \text { if } u \in \phi_{\lambda}(u) \text {. }
$$

By construction we have

$$
f(\lambda, u) \in \phi_{\lambda}(u)
$$

and

$$
\|f(\lambda, u)-u\| \leq(1+\delta \cdot \mu(T)) \cdot d\left(u, \phi_{\lambda}(u)\right) .
$$

We claim that $f(\lambda, u)$ is continuous.

Clearly, it is enough to check it at $(\lambda, u) \notin O$. Fix $(\lambda, u)$ such that

$$
u \in \phi_{\lambda}(u)
$$

and let $\lambda_{n} \rightarrow \lambda, u_{n} \rightarrow u$. Then by continuity

$$
\lim _{n \rightarrow \infty} d\left(u_{n}, \phi_{\lambda_{n}}\left(u_{n}\right)\right)=d\left(u, \phi_{\lambda}(u)\right)=0 .
$$

Therefore from (2.10) and (2.9) we obtain $f\left(\lambda_{n}, u_{n}\right) \rightarrow u=f(\lambda, u)$, proving the claim.

Set $g_{1}(\lambda, u)=f(\lambda, u)$ and by induction,

$$
g_{n+1}(\lambda, u)=f\left(\lambda, g_{n}(\lambda, u)\right) \text {. }
$$

Clearly each $g_{n}(\lambda, u)$ is continuous and by (2.9),

$$
g_{n+1}(\lambda, u) \in \phi_{\lambda}\left[g_{n}(\lambda, u)\right] \text {. }
$$

We shall show that $g_{n}$ converges locally uniformly to a continuous function $g(\lambda, u)$ satisfying (2.3) and (2.4).

Indeed, from (2.11) and (2.12) we see that

$$
\left\|g_{n+1}(\lambda, u)-g_{n}(\lambda, u)\right\| \leq(1+\delta \mu(T)) d\left(g_{n}(\lambda, u), \phi_{\lambda}\left(g_{n}(\lambda, u)\right)\right)
$$

$$
\begin{aligned}
& \leq(1+\delta \mu(T)) \cdot d_{H}\left(\phi_{\lambda}\left(g_{n}(\lambda, u)\right), \phi_{\lambda}\left(g_{n-1}(\lambda, u)\right)\right) \\
& \leq \alpha(1+\delta \mu(T))\left\|g_{n}(\lambda, u)-g_{n-1}(\lambda, u)\right\| .
\end{aligned}
$$

Therefore

$$
\left\|g_{n+1}(\lambda, u)-g_{n}(\lambda, u)\right\| \leq\left[\alpha(1+\delta \mu(T)]^{n} \cdot d\left(u, \phi_{\lambda}(u)\right)\right.
$$


Since the right-hand side of $(2.15)$ is locally bounded, $g_{n}(\lambda, u)$ converges locally uniformly. This gives the continuity of the map

$$
g(\lambda, u)=\lim _{n \rightarrow \infty} g_{n}(\lambda, u) .
$$

Moreover, for $u \in \phi_{\lambda}(u)$, we have $g(\lambda, u)=u$, for every $n$, since $g_{n}(\lambda, u)=$ $u$.

Passing to the limit in (2.12) we obtain

$$
g(\lambda, u) \in \phi_{\lambda}(g(\lambda, u)),
$$

proving (2.3).

Remark. For any $\varepsilon>0$ by choosing $\delta>0$ suitably small the previous construction yields a continuous family of retractions $g(\lambda, \cdot)$ such that

$$
\|g(\lambda, u)-u\| \leq((1+\varepsilon) /(1-\alpha))\left[d\left(u, \phi_{\lambda}(u)\right)+\varepsilon\right] .
$$

\section{APPLiCATIONS TO DIFFERENTIAL INCLUSIONS}

Let $E$ be a separable Banach space and consider a map $F:[0, T] \times E \rightarrow E$ with nonempty, closed, bounded values such that

(i) $F(\cdot, \cdot)$ is jointly measurable;

(ii) there exists an integrable $m \in L^{1}([0, T], R)$ such that for all $(x, y) \in$ $E$,

$$
d_{H}(F(t, x), F(t, y)) \leq m(t)|x-y| ;
$$

(iii) there exist $\alpha, \beta$ in $L^{1}([0, T], R)$ such that

$$
|F(t, x)| \leq \alpha(t)+\beta(t)|x| .
$$

For each $\xi \in E$ denote by $S_{\xi}$ the set of Carathéodory solutions of the Cauchy problem

$$
\dot{x} \in F(t, x), \quad x(0)=\xi
$$

and define

$$
\mathscr{F}_{\xi}=\left\{\dot{u}: u \in S_{\xi}\right\} \subset L^{1}([0, T], E) .
$$

Theorem 2. There exists a continuous $g: E \times L^{1} \rightarrow L^{1}$ such that

(i) $g(\xi, u) \in \mathscr{F}_{\xi}$ for all $u$;

(ii) $g(\xi, u)=u$ if $u \in \mathscr{F}_{\xi}$.

Proof. Consider the space $L^{1}$ of Bochner integrable functions $u:[0, T] \rightarrow E$ with the norm

$$
\|u\|_{*}=\int_{0}^{T}|u(t)| d \mu(t)
$$

where $\mu$ is the absolutely continuous measure on $[0, T]$ defined by:

$$
d \mu=e^{-2 r(t)} d t, \quad r(t)=\int_{0}^{t} m(s) d s .
$$


Set

$$
\phi_{\xi}(u)=\left\{v \in L^{1}: v(t) \in F\left(t, \xi+\int_{0}^{t} u(s) d s\right) \text { a.e. in }[0, T]\right\}
$$

and observe that $\mathscr{F}_{\xi}$ is precisely the set of fixed points of the map $u \rightarrow \phi_{\xi}(u)$. We claim that the map $(\xi, u) \rightarrow \phi_{\xi}(u)$ satisfies all hypotheses of Theorem 1 .

Indeed, to check $(2.1)$ given $v_{1} \in \phi_{\xi}\left(u_{1}\right)$ and $\varepsilon>0$, take $v_{2} \in \phi_{\xi}\left(u_{2}\right)$ such that, almost everywhere,

$$
\left|v_{1}(t)-v_{2}(t)\right|<d\left(v_{1}(t), F\left(t, \xi+\int_{0}^{t} u_{2}(s) d s\right)\right)+\varepsilon
$$

Then

$$
\begin{aligned}
\left\|v_{1}-v_{2}\right\|_{*}= & \int_{0}^{T} e^{-2 r(t)} \cdot\left|v_{1}(t)-v_{2}(t)\right| d t \\
\leq & \int_{0}^{T} e^{-2 r(t)} d\left(v_{1}(t), F\left(t, \xi+\int_{0}^{t} u_{2}(s) d s\right)\right) d t+\varepsilon \cdot \int_{0}^{t} e^{-2 r(t)} d t \\
\leq & \int_{0}^{T} e^{-2 r(t)} m(t) \cdot\left|\int_{0}^{T}\left(u_{2}(s)-u_{1}(s)\right) d s\right|+\varepsilon T \\
\leq & -\frac{1}{2} e^{-2 r(t)} \cdot \int_{0}^{T}\left|u_{2}(t)-u_{1}(t)\right| d t \\
& +\frac{1}{2} \int_{0}^{T} e^{-2 r}\left|u_{2}(t)-u_{1}(t)\right| d t+\varepsilon T \\
\leq & \frac{1}{2}\left\|u_{2}-u_{1}\right\|_{*}+\varepsilon T .
\end{aligned}
$$

Since $\varepsilon$ is arbitrarily small

$$
d\left(v_{1}, \phi_{\xi}\left(u_{1}\right)\right) \leq \frac{1}{2}\left\|u_{2}-u_{1}\right\|_{*}
$$

and, therefore,

$$
d_{H}\left(\phi_{\xi}\left(u_{1}\right), \phi_{\xi}\left(u_{2}\right)\right) \leq \frac{1}{2}\left\|u_{1}-u_{2}\right\|_{*} .
$$

Now Theorem 1 applied to the space $[0, T]$ with measure $d \mu=e^{-2 r(t)} d t$ gives the desired result.

\section{REFERENCES}

1. J. P. Aubin and A. Cellina, Differential inclusions, Springer-Verlag, Berlin, Heidelberg, and New York, 1984.

2. A. Bressan and G. Colombo, Extensions and selections of maps with decomposable values, Studia Math. 90 (1988), 69-86.

3. A. Cellina, On the set of solutions to Lipschitzian differential inclusions, Differential and Integral Equations 1 (1988), 495-500.

4. A. Cellina and A. Ornelas, Representation of the attainable set for Lipschitzean differential inclusions, Rocky Mountain J. Math. (to appear).

5. R. M. Colombo, A. Fryszkowski, T. Rzezukowski, and V. Staicu, Continuous selections of solutions sets of Lipschitzean differential inclusions, Funk. Ekv. (to appear). 
6. A. Fryszkowski, Continuous selections for a class of nonconvex multivalued maps, Studia Math. 76 (1983), 163-174.

7. F. Hiai and $\mathrm{H}$. Umegaki, Integrals, conditions expectations and martingales of multivalued functions, J. Multivariate Anal. 7 (1971), 149-182.

8. A. Ornelas, $A$ continuous version of the Filippov-Gronwall inequality for differential inclusions, Rend. Sem. Mat. Univ. Padova (to appear).

9. B. Ricceri, Une proprieté topologique de l'ensemble des points fixes d'une contraction multivoque à valeurs convexes, preprint.

10. K. Yosida, Functional analysis, 6th ed., Springer-Verlag, Berlin, 1980.

Scuola Internazionale Superiore di Studi Avanzati, Strada Costiera, 1134014 TRIESTE, ITALY 